\title{
FACILITIES FOR INDUSTRIAL CUSTOMERS AT THE DARESBURY SYNCHROTRON RADIATION SOURCE (SRS)
}

\author{
E. Towns-Andrews, E.J. MacLean*, CCLRC Daresbury Laboratory, Warrington, Cheshire, UK
}

\begin{abstract}
The Daresbury Synchrotron Radiation Source (SRS) [1] provides state-of-the-art analytical techniques from infrared to hard X-ray wavelengths. The unique characteristics of synchrotron radiation are ideal for analytical problems that require high spatial or temporal resolution or problems that are simply intractable using conventional instruments.

Here we outline the approach taken at the SRS towards industrial customers. We provide practical examples and case histories of how industrial processes have been improved in a diverse range of business sectors.
\end{abstract}

\section{INTRODUCTION}

An increasing number of large scale facilities exists worldwide, which have been traditionally used by universities and higher education institutions for pure research and development. In recognition of the needs of commercial customers, Daresbury Laboratory established DARTS (Daresbury Analytical Research and Technology Service) [2]. DARTS offers unique services which can be tailored to the needs of the customer, allowing access to synchrotron analytical facilities and also the significant expertise and knowledge of staff on site. The analytical portfolio offered by the facility encompasses imaging, spectroscopic and structural characterisation techniques. $D A R T S$ also has links to conventional analytical houses for standard analyses should these be required prior to use of the synchrotron. The nature of problems and issues solved by DARTS is varied and includes: investigations of product failure and non-conformance, manufacturing issues, basic R\&D and information used in expert witness legal cases.

\section{MARKETING STRATEGY}

As a means of focusing the marketing activity of DARTS, the UK Department of Trade \& Industry R\&D Scoreboard [3] was used to identify those key sectors with large spend in research and development. The areas identified were: pharmaceuticals and healthcare, consumer products and food, chemicals and specialities, oils and polymers and aerospace and defence. It should be noted that this does not preclude the option of doing work for organisations based in other market sectors.

DARTS has also ensured that all intellectual property rights arising out of a data collection on behalf of a customer belong to the customer. If a pharmaceutical company, for example, is developing a new drug it is vital for them that their intellectual property rights are irrefutable.
Our final strategy has been to appoint dedicated customer relations staff to ensure that the process of accessing the beamtime for industry is as straightforward as possible. By creating the one-stop shop there is no longer any need for customers to be dealing with several different departments at Daresbury for contracts, experimental risk assessments, accommodation etc.

\section{TECHNICAL APPLICATIONS}

As described above, DARTS is actively marketing to five key market sectors. Examples of the types of experiment done in each of these are now given.

\section{Pharmaceuticals \& Healthcare}

Pharmaceutical companies traditionally take a multidisciplinary approach to their research and hence use a variety of the techniques available on the SRS. Synchrotron X-ray diffraction has been used extensively in the structural studies of small molecules, which are potential drug candidates, and in their complexes with biological macromolecules. By using a combination of high-resolution powder diffraction, single crystal diffraction and protein crystallography, researchers from these companies have the potential for studying systems in depth at the same location.

A common technique used is single crystal X-ray diffraction for determining the molecular structure of drug molecules. Generally, single crystals can be grown that are large enough to use readily available laboratory-based $\mathrm{X}$-ray instruments. However, in some cases this is not possible and Station 9.8 on the SRS has opened up a new route to obtaining crystal structures [4]. By harnessing the power of the X-rays produced by the synchrotron, researchers can look at increasingly smaller crystals. The smallest crystal currently used for a successful structure determination is $5 \times 5 \times 5 \mu^{3}$ [5].

A recent example of a complex drug structure solved by DARTS was in work for Organon Laboratories [6]. The drug in question is a steroid, rocuronium bromide, which is used as a neuromuscular blocker during surgery under anaesthetic. The action of the drug is reversed by subsequent injection of a cyclodextrin that encapsulates the steroid molecule, thus reversing its blocking effect. Conventional analytical techniques had shown that a complex was formed but not the exact nature of it.

Due to the complexity of the structure involved and the small size of the crystals, the structure of the complex

\footnotetext{
*darts@dl.ac.uk
} 
could not be solved from data obtained using conventional lab equipment because the diffraction was too weak. Station 9.8 allowed sufficient data to be collected to solve the structure but, even once the data were collected, the structure solution was less than routine. However, by using the wide knowledge base at Daresbury, techniques from protein crystallography were used to solve the structure. The structure shown in Figure 1 illustrates how the cyclodextrin binds the rocuronium as predicted.

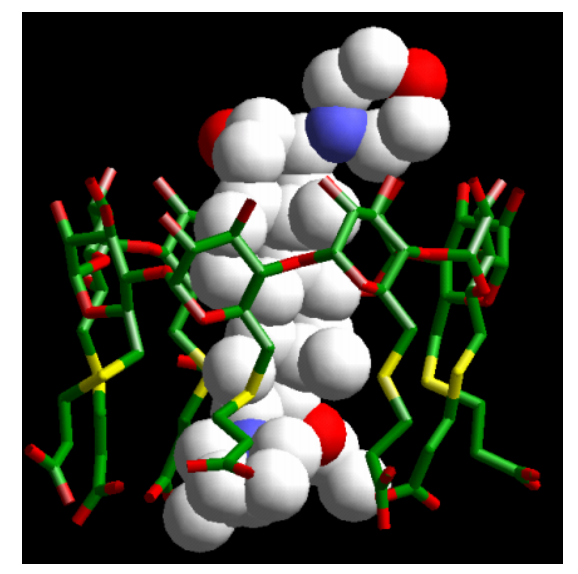

Figure 1: The complex of rocuronium bromide and a cyclodextrin

Preliminary trials have shown the cyclodextrin to reverse the effects of the neuromuscular blocker twice as fast as conventional treatments with none of the usual side effects. Human clinical trials are now taking place.

The second main area of interest for the pharmaceutical industry is determination of protein structure. Since many proteins are intrinsically associated with human health and disease, their structure can be key in developing new pharmaceuticals and drug therapies. The most common technique for obtaining precise information about the arrangement of atoms within a protein is X-ray crystallography. In many cases, conventional laboratory facilities cannot produce data of sufficient quality to resolve the structure of proteins. This is due to their complex characteristics such as low scattering power, poor ordering, crystal lifetime and susceptibility to radiation damage. Examples of non-proprietary work carried out at the SRS include, the anthrax lethal factor [7] and the structure of F1-ATPase [8] which was a key contributor to the Nobel Prize for Chemistry in 1997.

Where it is not possible to crystallise a particular protein, synchrotron UV circular dichroism (CD) can be used to determine secondary structure content. It has significant advantages over laboratory-based instruments including reduced data collection times, higher signal to noise ratios and extended wavelength resolution. Perhaps more importantly, CD can be used to study dynamic processes such as protein folding $[9,10]$ and misfolding the basis of diseases such as Alzheimer's and bovine spongiform encephalitis (BSE). For pharmaceutical companies the opportunity to study drug binding in real time and effects on protein secondary structure offers deeper understanding of potential drug candidates.

\section{Chemicals \& Specialities}

The use of pigments is widespread and includes coating technology, paints, inks and plastic coloration as well as more sophisticated technologies such as new information storage systems.

An important contributor to the colour of a pigment is the arrangement of the molecules within the pigment itself and, hence, to fully understand the colour properties of a pigment it is vital to know the crystal structure. Since a necessary property of a useful pigment is its small particle size, traditional approaches to obtaining crystal structures are not always possible. This is where the properties of synchrotron radiation can be exploited.

Solution of complex structures from powder diffraction data is a relatively new technique compared with single crystal structure solution. While it is possible to solve structures from powder diffraction methods using data collected on standard laboratory based diffractometers, the low symmetry of many organic materials can make analysis of the patterns troublesome due to severe peak overlap. The high resolution data obtainable using the SRS can sometimes overcome this problem.

One example of a pigment material studied using powder diffraction is DPP-Boc, a derivative of a class of pigments known as DPPs [11]. These are a relatively new class of organic pigments developed in the 1980s and 1990 s by Ciba Specialty Chemicals. DPP pigments have found wide-ranging commercial use because of the range of colours possible by simply varying different substituent groups within the molecule. The structure of DPP-Boc was intractable from laboratory data, as the powder diffraction pattern could not be indexed. However, the higher resolution data from the synchrotron allowed successful indexing and subsequent structure solution from powder data using the Monte Carlo method [12].

Whilst powder diffraction is an important technique for investigating long range order in systems such as the pigments described above, other customers in this sector are more interested in the ability to study local order in development of their products. Understanding the near neighbour environment of active sites in catalytic systems is important and X-ray absorption spectroscopy is routinely used to study systems such as zeolites and electrode surfaces.

The use of in situ combined techniques for observing development of long range and short range order in real time has proved very attractive for understanding catalyst preparation. For example, non-proprietary research into iron phosphate catalysts has successfully used combined $\mathrm{X}$-ray diffraction and quick scanning X-ray absorption spectroscopy at the SRS [13]. 


\section{Aerospace \& Defence}

Non-destructive testing of materials is an area of huge importance to industries such as automotive and aerospace. The non-conformance of materials to required quality and/or safety specifications can lead to large scale manufacturing production lines being brought to a halt while the problem is resolved. Representatives from these industries have employed the rapid turn-round service via DARTS to investigate component failures using a variety of specialist techniques. Novel X-ray imaging, surface and diffraction methods allow nondestructive testing of specimens which ensure that any observed effects have not been introduced during sample preparation. The versatility of these methods can be extended to many types of materials including metals, synthetic polymers and natural products.

Recent work at the SRS [14] has shown that fast residual stress mapping can be achieved in deformed plate materials using energy-dispersive transmission X-ray diffraction. Discrimination by energy rather than angle allows in situ measurements to be made without having to consider complications inherent in angle scanning such as low penetration, variable sample path lengths and awkward sample shapes. By using orthogonal scanning, the results have shown that a spatial resolution may be achieved of $100 \mu \mathrm{m}$ perpendicular to and of $1 \mathrm{~mm}$ along the beam for a number of different materials including a $6 \times 3 \times 100 \mathrm{~mm}$ two-phase Ti/Al/V aerospace alloy.

Having demonstrated the ability to optimise the SRS facility for this type of study, DARTS now offers fast residual stress mapping as a service for suitable materials.

\section{Consumer Products \& Food}

Companies in this sector manufacture products as diverse as food, cosmetics, detergents and healthcare products which must perform consistently to consumer expectations. Understanding product behaviour at the most basic level, for example the phase behaviour of products with regard to storage and processing conditions, is a crucial requirement for manufacturers. Some systems studied by DARTS include oils, detergents, foodstuffs, personal hygiene products and dermatological creams.

Crystallisation is one of the dominant techniques used by the chemical and food industries for particle separation and purification. However, there is still a limited understanding of the detailed mechanisms associated with the formation of crystalline and amorphous solids from liquid mother phases, be they melts or solutions. In chocolate production the solidification process requires careful control because it significantly influences both the rheological properties of chocolate such as viscosity and adhesion and the physical properties of the end product, which are vital in determining the acceptability of a product in the marketplace.
Collaborative work between Heriot-Watt University and the research and manufacturing arms of Cadbury Ltd. [15] has used the SRS for small angle X-ray scattering measurements using an environmental shear stress cell for in situ studies.

Results indicate that the effect of shearing on the crystallisation of cocoa butter is significant in determining which of six possible polymorphs develops. Shearing induces form $\mathrm{V}$ formation from the very outset, and this is the form needed to produce good quality chocolate with the desired rheological and physical properties. In the case of a stagnant run, form $\mathrm{V}$ is never present. Thus the production of good quality chocolate under these conditions would be virtually impossible.

This methodology could have many other applications well beyond confectionery, not merely in the food industry but throughout chemical industry generally, including pharmaceuticals, where the final physical form and properties of a product are governed by processing and production conditions.

Whilst synchrotron IR microspectroscopy is emerging as a powerful analytical tool for many industrial customers, the high spatial resolution and significantly improved signal to noise ratios achievable over conventional globar systems is being exploited by a number of DARTS customers in this particular sector. The chemical mapping of hair cross sections [16] clearly demonstrates the power of this technique for evaluating the effects of hair care products which are under development and also for forensic science. In addition, non-proprietary work into the study of extruded amylopectin-gelatin blends [17] and oil distribution in fried foods [18] has demonstrated the potential of this technique in the food industry

\section{Oils \& Polymers}

Work conducted in this sector covers a wide range of applications from development of novel catalysts to formulation of motor oils and production of packaging materials.

Polymer film manufacturer, UCB Films, requested evaluation of a coated polypropylene film used in food packaging, which degraded over a period of time resulting in increased opacity of the material when stored. The problem appeared to be related to the processing conditions under which the films had been manufactured.

DARTS utilised a multitechnique approach to this problem and employed grazing incidence X-ray diffraction (GIXD) and SAXS to investigate a number of polymer films from different processing batches. Figure 2 shows the GIXD data obtained from three samples. 


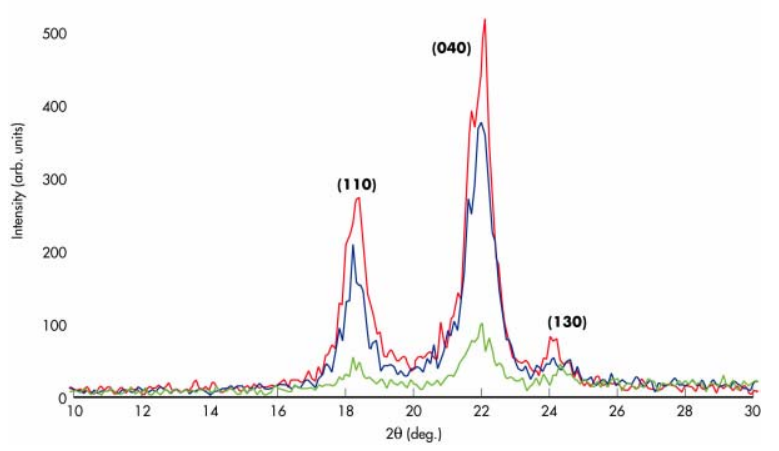

Figure 2 GIXD data of a series of polypropylene films

GIXD provided data from the $0.6 \mu \mathrm{m}$ co-polymer coating. Peaks observed corresponded exactly to those expected for polypropylene. Therefore, despite the presence of up to $10 \%$ ethylene in the copolymer, the molecular arrangement of polypropylene is being adopted in the crystallites of the coating layers. The peaks are sharper in the case of the clear films, implying a higher level of molecular order within the crystallites. SAXS data from the films indicated that the average particle size in the bulk material was approximately $200 \AA$ in each case. On-line processing facilities at the SRS have enabled other DARTS customers to study in real time the effects of varying processing conditions on the properties of polymer products $[19,20]$

In the manufacture of efficient and reliable engine oils it is important to understand and be able to control a multitude of complex chemical and physical processes. Examples of these processes include the interactions between different components of the engine oil and the effects of oil viscosity.

The role of the detergent in crank case lubricants is to provide a soluble base within the formulated engine oil and to neutralise acidic products which are introduced into the lubricant. Acidic products may originate from the blow-by gases produced during fuel combustion. Organic acids are also formed in situ in the crankcase through oxidation and degradation of the lubricant. If these acids are not rapidly neutralised, it may lead to corrosion within the crankcase.

Characterising the nature of the detergent additive, and hence its role in acid neutralisation, has proved difficult using standard analytical techniques. However, SAXS is ideally suited to this task since it provides a direct means of probing the structure of the detergent additive for different compositions of the oil base. Typically the calcium and magnesium detergent particles are $2050 \AA$ in size, a range that is easily accommodated by the SAXS method. In addition to providing information about particle size, SAXS also enables the particle shape and size distributions to be determined.

Infineum (a joint venture between Shell and Exxon) has used DARTS to access SAXS facilities at the SRS [21] to characterise detergent additives in motor oil samples. This has allowed quantification of process changes, control of rheological properties and performance characteristics of the finished product and prediction of the various interactions that can occur between detergent molecules and other additives in the finished oil.

\section{CONCLUSIONS}

The uniqueness of the DARTS service as a customerfacing support team is achieved by a dedicated, trained team of staff drawn from all areas of the Synchrotron Radiation Department. This has enabled the expansion of the portfolio of techniques and target sectors as described above. This approach allows UK and international industry to access the expertise available at Daresbury in a cost-effective manner - they do not all need to have synchrotron experts in their own labs. Of course, should industrial scientists wish to do their own experiments, this access mechanism is also available through DARTS. We offer a range of access levels from beamtime only to "you send the sample, we send the results". A combination of these levels has sometimes been the most convenient way for a company to use the service.

\section{REFERENCES}

[1] http://srs.ac.uk/srs

[2] http://www.darts.ac.uk

[3] http://www.innovation.gov.uk/projects/rd_scoreboard /introfr.html

[4] R.J. Cernik, W. Clegg, C.R.A. Catlow, G. BushnellWye, J.V. Flaherty, G.N. Greaves, I. Burrows, D.J. Taylor, S.J. Teat and M. Hamichi, J. Synch. Rad. 4 (1997) 279; W. Clegg, M.R.J. Elsegood, S.J. Teat, C. Redshaw and V.C. Gibson, J. Chem. Soc., Dalton Trans. (1998) 3037

[5] M. Wang, A. Mar and E.J. MacLean, J. Solid State Chem. in press (2003)

[6] A. Bom, M. Bradley, K. Cameron, J.K. Clark, J. van Egmond, H. Feilden, E.J. MacLean, A.W. Muir, R. Palin, D.C. Rees and M.-Q. Zhang, Angew. Chemie, Intl. Ed. Engl. 41 (2002) 266.

[7] A.D. Pannifer, T.Y. Wong, R. Schwarzenbacher, M. Renatus, C. Petosa, J. Bienkowska, D.B. Lacy, R.J. Collier, S. Park, S.H. Leppla, P. Hanna, and R.C. Liddington, Nature, 414 (2001) 229

[8] J-P. Abrahams, A.G.W. Leslie, R. Lutter and J.E. Walker Nature 370 (1994) 621

[9] B.A. Wallace, J. Synch. Rad. 7 (2000) 289

[10] D.T. Clarke, A.J. Doig, B.J. Stapeley and G.R. Jones, Proc. Natl. Acad. Sci. USA 96 (1999) 7232

[11]E.J. MacLean, M. Tremayne, B.M. Kariuki, K.D.M. Harris, A.F.M. Iqbal and Z. Hao, J. Chem. Soc., Perkin Trans. 2 (2000) 1513

[12] K.D.M. Harris, M. Tremayne, P. Lightfoot and P.G. Bruce J. Am. Chem. Soc. 116 (1994) 3543

[13] A.M. Beale and G. Sankar, J. Mater. Chem. 12 (2002) 3064

[14] A.M. Korsunsky, S.P. Collins, R.A. Owen, M.R. Daymond, S. Achtioui and K.E. James, J. Synch. Rad. 9 (2002) 77 
[15] S.D. MacMillan, K.J. Roberts, A. Rossi, M.A. Wells, M.C. Polgreen and I.H. Smith, Cryst. Growth Design 2 (2002) 221

[16]F. Briki, B. Busson, L. Kreplak, P. Dumas and J. Doucet, Cell. Mol. Biol 46 (2000) 1005

[17]Z. Mousia, I.A. Farhat, M. Pearson, M.A. Chesters and J.R. Mitchell, Biopolymers (Biospectroscopy) 62 (2001) 208

[18] P. Bouchon, P. Hollins, M. Pearson, D.L. Pyle and M.J. Tobin, Journal of Food Science 66 (2001) 918
[19]D.J. Blundell, G. Eeckhaut, W. Fuller, A. Mahendrasingam and C. Martin, Polymer 43 (2002) 5197

[20] N.J. Terrill, P.A. Fairclough, E. Towns-Andrews, B.U. Komanschek, R.J. Young and A.J. Ryan, Polymer 39 (1998) 238

[21] J. Galsworthy, S. Hammond and D. Hone, Current Opinion in Colloid and Interface Science 5 (2000) 27 